\title{
National Assessment of Oil and Gas Fact Sheet Assessment of Undiscovered Oil and Gas Resources of
the Southwestern Wyoming Province, 2002
}

Using a geology-based assessment methodology, the U.S. Geological Survey estimated a mean of 84.6 trillion cubic feet of undiscovered natural gas, a mean of 131 million barrels of undiscovered oil, and a mean of 2.6 billion barrels of undiscovered natural gas liquids in the Southwestern Wyoming Province.

\section{Introduction}

The U.S. Geological Survey (USGS) recently completed an assessment of the undiscovered oil and gas potential of the Southwestern Wyoming Province (fig. 1). The USGS Southwestern Wyoming Province for this assessment included the Green River Basin, Moxa arch, Hoback Basin, Sandy Bend arch, Rock Springs uplift, Great Divide Basin, Wamsutter arch, Washakie Basin, Cherokee Ridge, and the Sand Wash Basin. The assessment of the Southwestern Wyoming Province is based on the geologic elements of each Total Petroleum System (TPS) defined in the province, including hydrocarbon source rocks (source-rock maturation, hydrocarbon generation and migration), reservoir rocks (sequence stratigraphy and petrophysical properties), and hydrocarbon traps (trap formation and timing). Using this geologic framework, the USGS defined nine Total Petroleum Systems (TPS) and 23 Assessment Units (AU) within these TPSs and quantitatively estimated the undiscovered oil and gas resources within 21 of the 23 AUs (table 1).

\section{Resource Summary}

The USGS assessed undiscovered conventional oil and gas and undiscovered continuous (unconventional) oil and gas. The USGS estimated a mean of 84.6 trillion cubic feet of gas (TCFG), a mean of 131 million barrels of oil (MMBO), and a mean of 2.6 billion barrels of total natural gas liquids (BBNGL) in nine TPSs. Nearly all (97 percent, or $82.1 \mathrm{TCFG}$ ) of the undiscovered gas resource is continuous and distributed in six Total Petroleum Systems: Mowry Composite TPS (8.5 TCFG); Hilliard-BaxterMancos TPS (11.7 TCFG); Mesaverde TPS (25.8 TCFG); Mesaverde-Lance-Ft. Union Composite TPS (13.7 TCFG); Lewis TPS (13.5 TCFG); and Lance-Ft. Union Composite TPS (8.7 TCFG) (table 1). The remainder of the undiscovered gas is associated/dissolved gas in oil accumulations (0.13 TCFG) or is in conventional nonassociated gas accumulations (2.3 TCFG).

The Niobrara TPS is estimated to contain a mean of about 103 MMB of continuous oil, or about 79 percent of all undiscovered oil. The Phosphoria TPS contains a

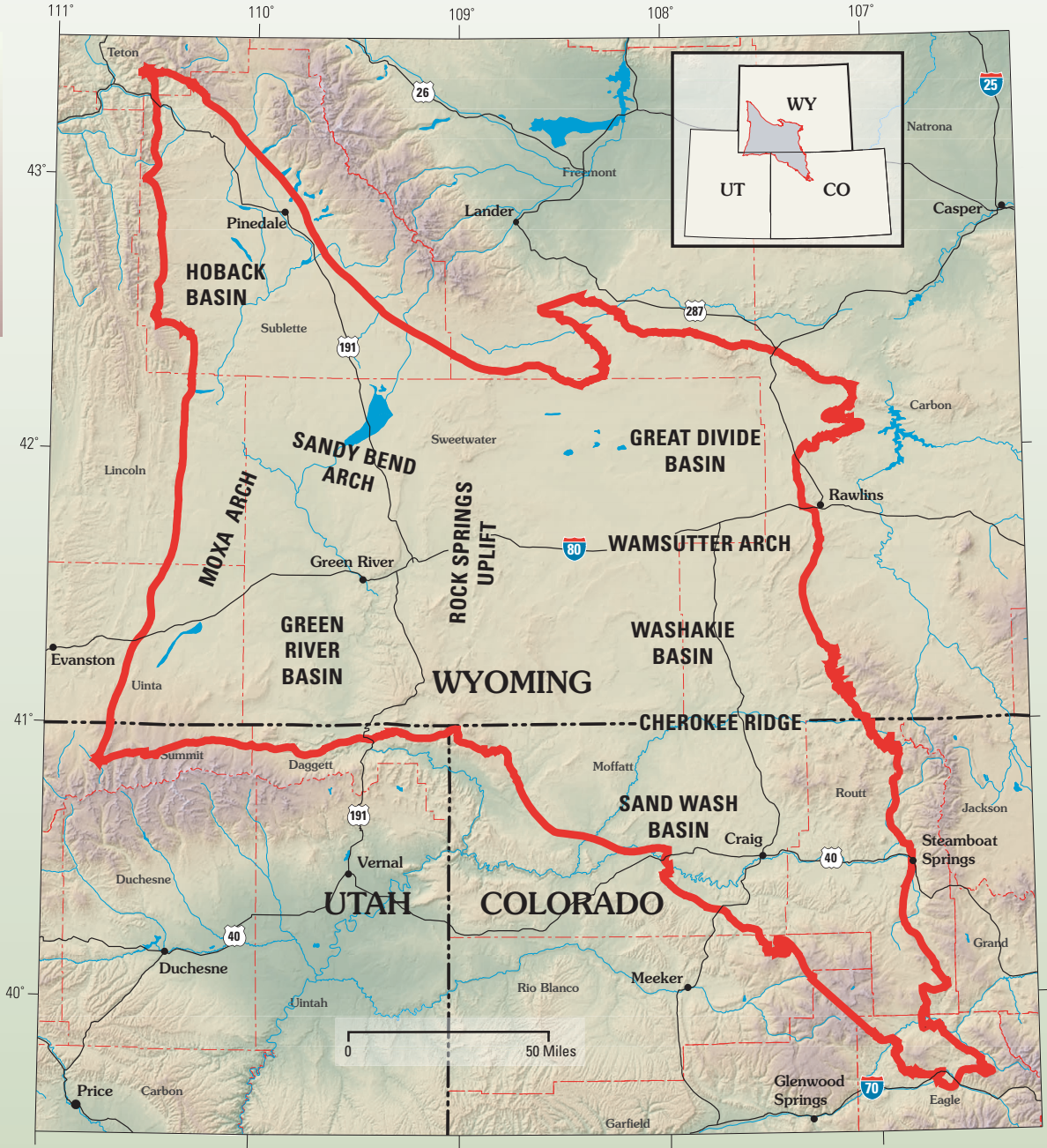

Figure 1. Southwestern Wyoming Province of southwestern Wyoming and northwestern Colorado.

mean of 16.6 MMBO, or about 12.5 percent of the undiscovered oil. The remainder of the undiscovered oil is in the Mowry TPS, the Mesaverde TPS, and the Mesaverde-Lance-Ft. Union Composite TPS (table 1). The Niobrara Continuous Gas AU and the Wasatch-Green River Continuous Gas AU were not quantitatively assessed in this study.

\section{For Further Information}

Supporting geologic studies of Total Petroleum Systems and Assessment Units, and reports on the methodology used in the Southwestern Wyoming Province assessment, are in progress. Assessment results are available at the USGS Central Energy Team website:

\section{http://energy.cr.usgs.gov/oilgas/noga/}

\section{Southwestern Wyoming Province Assessment Team:}

Mark Kirschbaum (Task Leader; mkirsch@usgs.gov), Thomas M. Finn, Robert D. Hettinger, Edward A. Johnson, Ronald C. Johnson, Joyce Kibler, Paul G. Lillis, Philip H. Nelson, Laura N.R. Roberts, Stephen B. Roberts, Ronald R. Charpentier, Troy A. Cook, Robert A. Crovelli, Timothy R. Klett, Richard M. Pollastro, and Christopher J. Schenk. 
Table 1. Southwestern Wyoming Province assessment results.

[MMBO, million barrels of oil; BCFG, billion cubic feet of gas; MMBNGL, million barrels of natural gas liquids. Results shown are fully risked estimates. For gas fields, all liquids are included under the NGL (natural gas liquids) category. F95 denotes a 95-percent chance of at least the amount tabulated. Other fractiles are defined similarly. Fractiles are additive only under the assumption of perfect positive correlation. CBG, coal-bed gas. Shading indicates not applicable]

Total Petroleum Systems

(TPS)

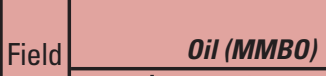

Total undiscovered resources

and Assessment Units (AU)

Field Gas (BCFG)

NGL (MMBNGL)

Phosphoria TPS

Sub-Cretaceous Conventional

Oil and Gas AU

Mowry Composite TPS

Mowry Conventional

Oil and Gas AU

\section{Hilliard-Baxter-Mancos TPS}

Hilliard-Baxter-Mancos

Conventional Oil and Gas AU

\begin{tabular}{|l|l|l|l|l|l|}
\hline Oil & 3.80 & 12.70 & 43.60 & 16.60 & \\
\hline Gas & & & & \\
\hline
\end{tabular}

\begin{tabular}{l|l|l|l|l|l|l|l} 
F95 & F50 & F5 & Mean & F95 & F50 & F5 & Mean
\end{tabular}

Mesaverde TPS

Mesaverde Conventional

Oil and Gas AU

\begin{tabular}{|l|r|r|r|r|r|r|r|r|r|r|r|r|}
\hline Oil & 1.70 & 5.70 & 14.80 & 6.60 & 2.70 & 9.40 & 25.90 & 11.20 & 0.30 & 1.30 & 3.70 & 1.60 \\
\hline Gas & & & & & 85.80 & 196.30 & 301.40 & 195.10 & 1.60 & 3.80 & 6.70 & 3.90 \\
\hline
\end{tabular}

Mesaverde-Lance-Ft. Union Composite TPS

Mesaverde-Lance-Ft. Union

Conventional Oil and Gas AU

Gas

\begin{tabular}{|l|r|r|r|r|r|r|r|}
\hline 6.70 & 24.00 & 85.90 & 32.20 & 0.20 & 0.80 & 3.20 & 1.20 \\
\hline
\end{tabular}

\begin{tabular}{|l|l|l|l|l|l|l|l|}
\hline 206.20 & $1,069.00$ & $3,480.00$ & $1,350.70$ & 5.90 & 31.20 & 107.20 & 40.60 \\
\hline
\end{tabular}

\section{Lewis TPS}

Lewis Conventiona

Oil and Gas AU

\begin{tabular}{|l|l|l|l|l|r|r|r|r|r|r|r|r|}
\hline Oil & 0.90 & 2.10 & 4.00 & 2.30 & 7.40 & 17.30 & 34.80 & 18.80 & 0.30 & 0.60 & 1.40 & 0.70 \\
\hline Gas & & & & & 13.40 & 34.00 & 69.40 & 36.90 & 0.10 & 0.40 & 0.90 & 0.40 \\
\hline
\end{tabular}

Lance-Ft. Union Composite TPS

Lance-Ft. Union Conventional

Oil and Gas AU

Total Conventional

Resources

\begin{tabular}{|l|l|l|l|l|}
\hline Oil & 0.90 & 2.10 & 4.00 & 2.30 \\
\hline Gas & & & & \\
\hline
\end{tabular}

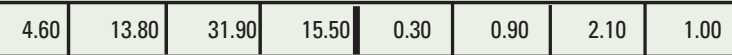

Mowry Composite TPS

\begin{tabular}{|l|l|l|l|l|l|l|}
\hline Gas & & & & & & \\
\hline
\end{tabular}

\begin{tabular}{r|r|r|r|r|r|r|r|}
\hline 3.80 & 9.10 & 18.30 & 9.80 & 0.20 & 0.40 & 0.90 & 0.40 \\
\hline 101.40 & 297.70 & 558.80 & 310.40 & 4.20 & 13.00 & 26.90 & 14.00 \\
\hline
\end{tabular}

Mowry Continuous Gas AU

\begin{tabular}{|l|l|l|l}
\hline Gas & \\
\hline &
\end{tabular}

7.30

\begin{tabular}{l|l|l}
22.60 & 66.40 & 27.80
\end{tabular}

Gas

Niobrara TPS

Niobrara Continuous Oil AU

Niobrara Continuous Gas AU

\begin{tabular}{|l|l|l|l|l}
\hline Oil & 66.90 & 100.50 & 151.00 & 103.60 \\
\hline Gas & \multicolumn{1}{|l}{} \\
\hline
\end{tabular}

Hilliard-Baxter-Mancos TPS

Hilliard-Baxter-Mancos

Continuous Gas AU

Gas

Gas

Mesaverde TPS

Almond Continuous Gas AU

Rock Springs-Ericson

Continuous Gas AU

Mesaverde Coal-Bed Gas AU

\begin{tabular}{|l|l|l|l|l|}
\hline Gas & & & & \\
\hline Gas & & & & \\
\hline cBG & & & & \\
\hline
\end{tabular}

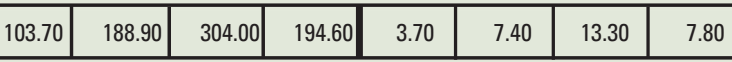

Mesaverde-Lance-Ft. Union Composite TPS

Mesaverde-Lance-Ft. Union

Continuous Gas AU

Mesaverde Coal-Bed Gas AU

Ft. Union Coal-Bed Gas AU

\begin{tabular}{|l|l|l|l|l|}
\hline Gas & & & & \\
\hline CBG & & & & \\
\hline CBG & & & & \\
\hline
\end{tabular}

\begin{tabular}{|l|l|l|l|l|l|l|l|}
\hline $8,320.10$ & $13,122.00$ & $20,695.40$ & $13,635.20$ & 329.20 & 578.60 & $1,016.90$ & 613.60 \\
\hline
\end{tabular}

\begin{tabular}{|r|r|r|r|r|r|r|r|}
\hline 13.70 & 25.40 & 47.30 & 27.30 & 0.00 & 0.00 & 0.00 & 0.00 \\
\hline
\end{tabular}

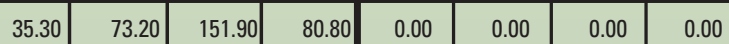

Lewis TPS

Lewis Continuous Gas AU

Gas

Lance-Ft. Union Composite TPS

Lance-Ft. Union Continuous Gas AU Gas

Lance Coal-Bed Gas AU

Ft. Union Coal-Bed Gas AU

CBG

CBG

Wasatch-Green River Composite TPS

Wasatch-Green River

Continuous Gas AU

Wasatch-Green River

Coal-Bed Gas AU

Total Continuous

Resources

Total Undiscovered

Oil and Gas Resources

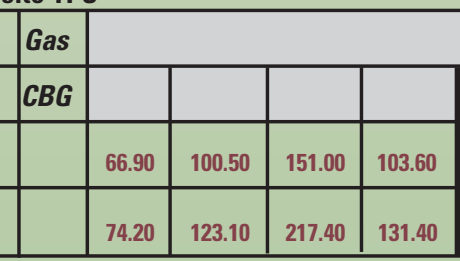

Not quantitatively assessed

\begin{tabular}{|l|r|r|r|r|l|l|l|}
\hline 27.80 & 58.40 & 122.60 & 64.70 & 0.00 & 0.00 & 0.00 & 0.00 \\
\hline
\end{tabular}

\begin{tabular}{|r|r|r|r|r|r|r|r|}
\hline $4,450.60$ & $7,255.80$ & $11,829.10$ & $7,583.30$ & 39.40 & 71.10 & 128.40 & 75.80 \\
\hline 78.20 & 152.00 & 295.50 & 165.00 & 0.00 & 0.00 & 0.00 & 0.00 \\
\hline 513.90 & 891.20 & $1,545.40$ & 942.50 & 0.00 & 0.00 & 0.00 & 0.00 \\
\hline
\end{tabular}

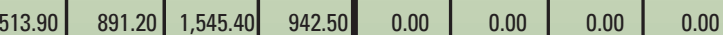

Not quantitatively assessed

\begin{tabular}{|l|l|l|l|l|l|l|l|}
\hline $4,895.10$ & $10,542.00$ & $22,703.40$ & $11,753.20$ & 286.50 & 661.10 & $1,525.20$ & 752.20
\end{tabular}

\begin{tabular}{|l|l|l|l|l|l|l|l|}
\hline $10,013.50$ & $13,166.10$ & $17,311.30$ & $13,349.70$ & 113.50 & 190.60 & 319.90 & 200.20 \\
\hline
\end{tabular}

\begin{tabular}{|l|l|l|l|l|l|l|l|}
$8,768.90$ & $11,962.80$ & $16,320.00$ & $12,178.00$ & 89.20 & 140.70 & 221.70 & 146.10 \\
\hline
\end{tabular}

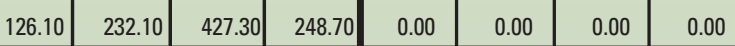

\begin{tabular}{ll}
74.20 & 123.10 \\
\hline
\end{tabular}

131.40

\begin{tabular}{|l|l|l|l|l|l|l|l|}
\hline $52,788.90$ & $79,134.80$ & $121,830.90$ & $82,169.10$ & $1,275.60$ & $2,326.10$ & $4,335.20$ & $2,503.90$ \\
\hline
\end{tabular}

\begin{tabular}{|l|l|l|l|l|l|l|l|}
$553,399.60$ & $81,223.50$ & $127,207.20$ & $84,589.90$ & $1,293.10$ & $2,388.10$ & $4,506.50$ & $2,578.00$ \\
\hline
\end{tabular} 